\title{
THE CUSPED HYPERBOLIC 3-ORBIFOLD OF MINIMUM VOLUME
}

\author{
BY ROBERT MEYERHOFF ${ }^{1}$
}

An orbifold is a space locally modelled on $\mathbf{R}^{n}$ modulo a finite group action. We will restrict our attention to complete orientable hyperbolic 3-orbifolds $Q$; thus, we can think of $Q$ as $H^{3} / \Gamma$, where $\Gamma$ is a discrete subgroup of Isom $_{+}\left(H^{3}\right)$, the orientation-preserving isometries of hyperbolic 3-space. An orientable hyperbolic 3-manifold corresponds to a discrete, torsion-free subgroup of Isom $_{+}\left(H^{3}\right)$. We will work in the upper-half-space model $H^{3}$ of hyperbolic 3-space, in which case $\operatorname{PGL}(2, \mathrm{C})$ acts as isometries on $H^{3}$ by extending the action of $P G L(2, \mathbf{C})$ on the Riemann sphere (boundary of $H^{3}$ ) to $H^{3}$. If the discrete group $\Gamma$ corresponding to $Q$ has parabolic elements, then $Q$ is said to be cusped. (For more details on this paragraph see [T, Chapter 13].)

Unless otherwise stated, we will assume all manifolds and orbifolds are orientable. Mostow's theorem implies that a complete, hyperbolic structure of finite volume on a 3-orbifold is unique. Consequently, hyperbolic volume is a topological invariant for orbifolds admitting such structures. Jørgensen and Thurston proved (see $[\mathbf{T}, \S 6.6]$ ) that the set of volumes of complete hyperbolic 3-manifolds is well-ordered and of order type $\omega^{\omega}$. In particular, there is a complete hyperbolic 3-manifold of minimum volume $V_{1}$ among all complete hyperbolic 3-manifolds and a cusped hyperbolic 3-manifold of minimum volume $V_{\omega}$. Further, all volumes of closed manifolds are isolated, while volumes of cusped manifolds are limits from below (thus the notation $V_{\omega}$ ).

Modifying the proofs in the Jørgensen-Thurston theory yields similar results for complete hyperbolic 3-orbifolds (but see the remark at the end of this paper). In particular, there is a hyperbolic 3-orbifold of minimum volume, and a cusped hyperbolic 3 -orbifold of minimum volume. We prove

THEOREM. Let $Q_{1}=H^{3} / \Gamma_{1}$ where $\Gamma_{1}=P G L\left(2, O_{3}\right)$ and $O_{3}=$ ring of integers in $Q(\sqrt{-3})$. The orbifold $Q_{1}$ has minimum volume among all orientable cusped hyperbolic 9-orbifolds.

Note. $Q_{1}$ is the orientable double-cover of the (nonorientable) tetrahedral orbifold with Coxeter diagram $\ldots$ (see [ $\mathbf{T}$, Theorem 13.5.4] and [H, $\S 1]$ ). This tetrahedral orbifold has fundamental domain $1 / 24$ of the ideal regular hyperbolic tetrahedron (use the symmetries). In particular, $Q_{1}$ has a cusp and its volume is $1 / 12$ the volume of the ideal regular tetrahedron $T$, i.e. $\operatorname{vol}\left(Q_{1}\right)=V / 12 \approx 0.0846$, where $V=\operatorname{vol}(T)$.

Received by the editors October 2, 1984.

1980 Mathematics Subject Classification. Primary 20H10, 51M25.

${ }^{1}$ Research partially supported by NSF grant MCS 8201827 .

(C)1985 American Mathematical Society $0273-0979 / 85 \$ 1.00+\$ .25$ per page 
PROOF (OF THEOREM). In Parts I and II of the proof we will get a lower bound for the volume of $H^{3} / \Gamma$ for arbitrary cusped discrete $\Gamma$.

\section{PART I: VOLUME CONTRIBUTIONS OF CUSPED NEIGHBORHOODS IN $H^{3} / \Gamma$.}

MANIFOLD CASE (i.e., $\Gamma$ such that $H^{3} / \Gamma$ is a manifold with a cusp): We can assume (using a suitable conjugation) that the cusp corresponds to the point at $\infty$ in $H^{3}$, and that the parabolic transformation $z \mapsto z+1$ is the "shortest" element in $\Gamma_{\infty}$, the stabilizer of $\infty$ in $\Gamma\left(\Gamma_{\infty}\right.$ has no hyperbolic elements; see [Be, Theorem 5.1.2]). Construct the horoball $C_{\infty}$, centered at $\infty$, for which $\Gamma_{\infty}$ has minimum translation length one (in the Euclidean metric) on the horosphere boundary of $C_{\infty}$. Our set-up has been rigged so that $C_{\infty}=\{(x, y, t): t \geq 1\}$. Construct such "length one" cusp neighborhoods at all parabolic fixed points (for some element of $\Gamma$ ). It is a standard fact (see [Be, Theorem 5.4.4]) that all such cusp neighborhoods are disjoint. Thus $C_{\infty} / \Gamma_{\infty}$ is an embedded "cusp neighborhood" in $M=H^{3} / \Gamma$.

What is the volume of $C_{\infty} / \Gamma_{\infty}$ ? If $z \mapsto z+1$ is the "shortest element" in $\Gamma_{\infty}$, then any other element $z \mapsto z+w$ in $\Gamma_{\infty}$ must have $|w| \geq 1$ and $|\operatorname{Im}(w)| \geq \sqrt{3} / 2$. Thus, we can compute $\operatorname{vol}\left(C_{\infty} / \Gamma_{\infty}\right) \geq \sqrt{3} / 4$ (see [M1, §5]).

ORBIFOLD CASE. The only additional complication from the manifold case is that $\Gamma_{\infty}$ may include elliptic elements. If so, then the elliptic and parabolic elements comprising $\Gamma_{\infty}$ act as rigid motions on the (Euclidean) horosphere at height 1 in $H^{3}$. Thus, we need only study the oriented wall-paper groups to understand the effect of the elliptic elements on the volume estimate for $C_{\infty} / \Gamma_{\infty}$. There are 5 such wall-paper groups, and the worst case reduces volume by a factor of 6 .

The cusp neighborhoods contribute at least $\sqrt{3} / 24$ to the volume of a complete orientable cusped hyperbolic 3-orbifold.

PART II: VOLUME CONTRIBUTIONS OUTSIDE THE CUSP NEIGHBORHOODS. By Part I, we have some control over the size of a cusped neighborhood. However, this cusp neighborhood is only a portion of the fundamental domain for $\Gamma$. Can we gain some control over the size of the fundamental domain outside of the cusp neighborhood? Yes, by sphere-packing. First, we fix a particular fundamental domain $D$ for $\Gamma$ : Let $D_{\infty}=\left\{p \in H^{3}: p\right.$ is closer to $C_{\infty}$ than to any conjugate (under $\Gamma$ ) of $\left.C_{\infty}\right\}$. Then we take $D$ to be a fundamental domain for the action of $\Gamma_{\infty}$ on $D_{\infty}$.

Next, consider 4 horospheres in $H^{3}$, each touching all the others. Their centers (points of tangency with $\partial H^{3}$ ) will determine an ideal regular tetrahedron $T$. Let $B$ be the union of the 4 horoballs bounded by the 4 horospheres. Böröczky's theorem (see [B, Theorem 4]) says that this is, in some sense, the densest packing of horospheres in hyperbolic 3-space. In terms of $C_{\infty}$ and $D$, Böröczky's theorem implies that $\operatorname{vol}\left(C_{\infty} \cap D\right) / \operatorname{vol}(D) \leq \operatorname{vol}(B \cap T) / \operatorname{vol}(T)=$ $4(\sqrt{3} / 8) / V=\sqrt{3} / 2 V$ (for more details, see $[\mathbf{M 2}]$ ).

$$
\begin{aligned}
& \text { Thus, } \operatorname{vol}\left(H^{3} / \Gamma\right)=\operatorname{vol}(D) \geq \operatorname{vol}\left(C_{\infty} \cap D\right) /(\sqrt{3} / 2 V) \geq \\
& (\sqrt{3} / 24)(2 V / \sqrt{3})=V / 12 .
\end{aligned}
$$


PART III: SUMmaRY. As mentioned above, the orbifold $Q_{1}$ has a cusp and has volume $V / 12$. Parts I and II tell us that all cusped orbifolds have volume at least $V / 12$. Thus $Q_{1}$ realizes the minimum volume and it is $V / 12 \approx$ 0.0846 .

REMARK. There are cusped orbifolds on which Dehn surgery cannot be performed. Consequently, unlike the manifold case, there are cusped hyperbolic 3-orbifolds whose volumes are isolated $-Q_{1}$ is such an orbifold. The question of finding "the least limiting orbifold" remains open.

ACKNOWLEDGEMENTS. I thank John Smillie for a helpful conversation. Also, most of this research was done at the Institute for Advanced Study; I thank the Institute for its hospitality.

\section{BIBLIOGRAPHY}

[Be] A. Beardon, The geometry of discrete groups, Springer-Verlag, New York, 1983.

[B] K. Böröczky, Packing of spheres in spaces of constant curvature, Acta Math. Acad. Sci. Hungar. 32 (1978), 243-261.

[H] A. Hatcher, Hyperbolic structures of arithmetic type on some link complements, J. London Math. Soc (2) 27 (1983), 345-355.

[M1] R. Meyerhoff, $A$ lower bound for the volume of hyperbolic 3-manifolds, preprint.

[M2] _ , Sphere-packing and volume in hyperbolic 3-space, preprint.

[T] W. Thurston, The geometry and topology of 3-manifolds, Princeton Univ. preprint 1978.

Department of Mathematics, Michigan State University, East LansING, MICHIGAN 48824

Current address: Department of Mathematics, Boston University, Boston, Massachusetts 02215 